\title{
Comparison dimension cues in delayed matching by pigeons: The effect of delay interval and cue-presentation method
}

\author{
ANGELO SANTI and CORINNE DONALD \\ Wilfrid Laurier University, Waterloo, Ontario, Canada
}

\begin{abstract}
Pigeons were trained on a delayed matching task in which a cue (a circle or a triangle) superimposed on the sample stimulus (red or green) signaled whether the test consisted of color or linetilt comparison stimuli. During probe testing sessions, accuracy was assessed on correctly cued (CC), incorrectly cued (IC), and no-cue (NC) trials. In Experiment 1, accuracy was lower on IC trials than on CC or NC trials. This cuing effect remained constant as the delay interval was increased. In Experiment 2, the cuing effect occurred only when the cue was superimposed on the sample. Experiment 3 revealed that presample and postsample presentation of the cue was ineffective in promoting a cuing effect. The results were inconsistent with a dual coding model that postulates that both prospective and retrospective coding occurs in this task. The data suggested that the cues and sample stimuli were processed and perceived as unitary configurations. Comparison cues do not function independently of the sample when they are superimposed on it during training.
\end{abstract}

Researchers in the area of animal cognition have developed a variety of research approaches in an attempt to determine whether the memory code in a delayed matchingto-sample task is a prospective representation of the correct comparison stimulus to respond to, or a retrospective representation of, sample stimulus attributes (Cohen, Galgan, \& Fuerst, 1986; Grant, 1982; Honig \& Thompson, 1982; Kraemer \& Roberts, 1984; Santi \& Roberts, 1985; Urcuioli \& Zentall, 1986). One approach to addressing this issue has been to provide pigeons with a cue signaling the comparison dimension. Stonebraker and Rilling (1984) found that providing the birds with an incorrect cue for the comparison dimension resulted in a significant drop in accuracy relative to correctly cuing the dimension. Although they interpreted this result as supportive of a prospective processing hypothesis, others have noted that the results are also consistent with a retrospective processing hypothesis.

Santi, Musgrave, and Bradford (1988) attempted to distinguish between these two coding hypotheses by examining performance on incorrectly cued (IC) trials relative to no-cue (NC) trials. A prospective hypothesis predicts lower accuracy on IC trials than on NC trials. A retrospective hypothesis, on the other hand, suggests equivalent performance on IC and NC trials. Two methods of cue presentation were studied in Santi et al. (1988). The simultaneous compound condition, in which the cue was superimposed on the sample stimulus, produced the largest cu-

This research was supported by Grant A6378 from the Natural Sciences and Engineering Research Council of Canada to Angelo Santi. The authors would like to thank Elizabeth Layne, Charlotte McCrae, Eve Atkinson, Steve Bridson, and Paulette West for their technical assistance. Reprints may be obtained from Angelo Santi, Department of Psychology, Wilfrid Laurier University, Waterloo, Ont. N2L 3C5, Canada. ing effect. Accuracy on IC trials was significantly lower than on either correctly cued (CC) or NC trials.

On the basis of the Santi et al. (1988) data, it was evident that the pigeons were not engaged exclusively in prospective coding of the sample stimuli. If they were, a much larger difference in performance between IC and NC trials would be expected, with accuracy on IC trials being much closer to chance levels. Consequently, Santi et al. speculated that the pigeons were coding samples both prospectively and retrospectively on each trial, with the experimental procedure biasing the birds to rehearse and respond on the basis of the prospective code. In the absence of the prospective code, on some portion of the IC trials the birds could respond on the basis of the retrospective code, but this code would have received less rehearsal than the prospective code. According to this dual coding model, accuracy on IC trials should be lower than on either CC or NC trials, but it should be above chance. This model also leads to the prediction that increases in the delay interval (DI) duration should affect IC trial accuracy more than CC or NC trial accuracy. On IC trials, the longer the bird spends rehearsing the inappropriate prospective code, the less likely it is that the retrospective code will be available at the end of the DI. In Experiment 1 , this prediction was tested and an attempt was made to increase the magnitude of the cuing effect by having the cues signal different probabilities of reinforcement on the two comparison dimensions.

\section{EXPERIMENT 1}

\section{Method \\ Subjects. Eight White Carneaux pigeons, maintained at approximately $80 \%$ of their free-feeding weight and housed individually with constant}


access to grit and water, served as subjects. All birds had served in a previous study of comparison dimension cuing (Santi et al., 1988).

Apparatus. Four Coulbourn modular operant test cages (Model E10-10), housed individually in isolation cubicles (Model E10-20), were used. Each cubicle was equipped with a ventilation fan and baffled air intake and exhaust system. Each test cage was equipped with three horizontally aligned, clear plastic keys behind which projectors could display stimuli (red or green field, vertical or horizontal white line on a black background, white circle or white triangle on a black background, or black dot on a white background) onto a frosted rear-projection screen (Coulbourn Model E21-18). Directly below the center key was a $5.7 \times 5 \mathrm{~cm}$ opening that provided access to a hopper filled with mixed grain (Coulbourn Model E14-10). All experimental events and response measures were arranged and recorded by a microcomputer system located in an adjacent room.

Procedure. All birds had had extensive previous training in a delayed matching task in which colors had served as sample stimuli and cues consisting of a circle or a triangle signaled whether the test consisted of color or line-tilt comparison stimuli. Each trial began with illumination of the center key by a warning stimulus (a black dot on a white background). A peck to the warning stimulus was followed by the presentation of the sample stimulus (red or green) and a superimposed cue (circle or triangle). The sample was terminated with the first keypeck after $5 \mathrm{sec}$ had elapsed; however, the cue continued to be presented on the center key during the 1-sec DI. At the end of the DI, the comparison stimuli were presented on the side keys and the cue was presented on all three keys. The cue was presented on all three keys during the comparison presentation phase of a trial in order to increase the strength of the association between the cues and the comparison dimensions.

For the 4 birds in the nondifferential reinforcement group, the cues signaled different comparison dimensions only. The probability of reinforcement was the same for both test dimensions. For 3 birds in this group, the circle signaled line comparisons and the triangle signaled color comparisons. This was reversed for the remaining bird in this group. Three out of every five correct responses to either color or line comparisons were reinforced in this group (probability of reinforcement $=.6$ ). For the 4 birds in the differential reinforcement group, the cues signaled different test dimensions as well as different probabilities of reinforcement on these dimensions. For 2 of the birds, the probability of reinforcement was 1.0 for correct responses to color comparisons but only .2 for correct responses to line comparisons. This was reversed for the other 2 birds in this group. For 2 birds, the circle signaled line comparisons and the triangle signaled color comparisons. This was reversed for the remaining 2 birds in this group. A correct response resulted in termination of the comparison stimuli and cues and, on reinforced trials, in $3 \mathrm{sec}$ of access to mixed grain. Incorrect responses or correct responses on nonreinforced trials resulted in termination of comparison stimuli and cues and a 3-sec blackout. Following either reinforcement or blackout, an intertrial interval (ITI) of $10 \mathrm{sec}$ was spent in darkness.

Baseline sessions prior to testing consisted of 96 trials ( 48 color tests and 48 line tests). On the last three baseline sessions prior to testing, all birds averaged $83 \%$ correct or higher. Each test session consisted of $120 \mathrm{CC}$ trials and 24 probe trials. The 24 probe trials consisted of $8 \mathrm{CC}$ trials, 8 IC trials, and $8 \mathrm{NC}$ trials. A single probe trial occurred randomly within each block of $5 \mathrm{CC}$ trials. During test sessions, the DI was either $1 \mathrm{sec}$ or $5 \mathrm{sec}$. Except for NC trials, the cues were presented throughout the DI. The DI duration was individually randomized for each bird in a 2-day block. A total of 16 test sessions were given, 8 at each DI.

\section{Results and Discussion}

The data obtained on probe trials were collapsed over test sessions and are shown in Figure 1. An analysis of variance revealed no significant effect of groups or interaction of groups with other factors. There was an effect of trial type $[F(2,12)=25.43, p<.001]$. A NewmanKeuls test revealed that accuracy was significantly lower on IC trials than on either CC or NC trials. Accuracy on CC and NC trials did not differ significantly. The data also revealed a statistically significant decline in accuracy as the DI was increased from 1 to $5 \sec [F(1,6)=7.07, p<.05]$.
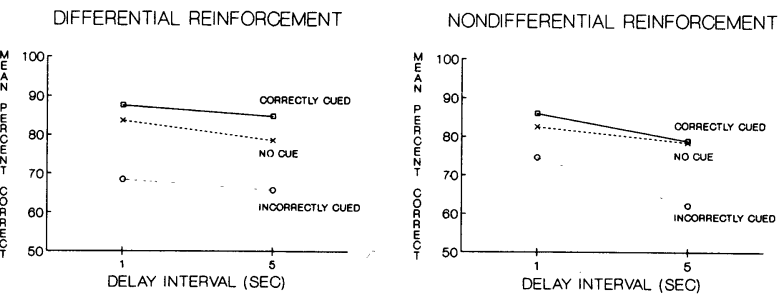

Figure 1. Mean percentage of correct matching responses during testing as a function of delay interval, trial type (correct, incorrect, or no cue), and reinforcement condition in Experiment 1.

However, there was no significant trial type $\times$ DI interaction $(F<1)$. We obtained similar results in an unpublished study in which baseline DIs were individually adjusted for each bird prior to testing. Testing was carried out at the baseline DI and at a DI 3 sec greater than the baseline delay. The effect of increasing the DI in this manner was as large on IC trials as it was on CC trials. The insensitivity of the cuing effect to DI duration is inconsistent with a dual coding model that postulates that the sample stimulus activates both a prospective and a retrospective code on each trial, with the retrospective code being rehearsed less than the prospective code. Grant and MacDonald (1990) have recently rejected dual coding interpretations of comparison cuing effects on the basis of similar results.

\section{EXPERIMENT 2}

The purpose of this experiment was to determine how different methods of cue presentation might affect the way in which sample information was processed.

\section{Method}

Subjects and Apparatus. The subjects and the apparatus were the same as in Experiment 1.

Procedure. Prior to the testing phase of this experiment, all birds received 13 sessions of baseline training. On each trial, a peck to the warning stimulus produced a sample stimulus (red or green) on the center key, with a cue (circle or triangle) superimposed on it. The first keypeck after $5 \mathrm{sec}$ terminated the sample stimulus, but the cue remained on during the DI. The DI was gradually increased from 1 to $4 \mathrm{sec}$ during the baseline training phase. The cue-comparison-dimension assignment for each bird was the same as that used in the previous experiment. In this study, the cue was not presented during the comparison presentation phase of a trial. Four birds received a high probability of reinforcement (1.0) for correct responses to color comparison stimuli and a low probability of reinforcement $(.2)$ for correct responses to line comparisons. This was reversed for the other 4 birds. A correct response terminated the comparison stimuli and on reinforced trials resulted in $3 \mathrm{sec}$ of access to mixed grain. Incorrect responses or correct responses on nonreinforced trials resulted in termination of comparison stimuli and a 3-sec blackout. An ITI of $10 \mathrm{sec}$ was spent in darkness. Baseline sessions consisted of 120 trials (60 color and 60 line). Averaged matching accuracy over the last 3 baseline sessions with a DI of 4 sec ranged from $74 \%$ to $94 \%$ for individual birds.

During the testing phase, four different methods of cue presentation were assessed. In the SUPFULL condition, the cue was superimposed on the sample and presented for the full duration of the DI $(4 \mathrm{sec})$. In the SUPHALF condition, the cue was superimposed on the sample and remained on for half of the DI $(2 \mathrm{sec})$. In the SUPNO condition, the cue was superimposed on the sample but was not presented during the DI. In the SUCCFULL condition, the cue was presented after termination 
of the sample for the full duration of the DI. During testing, the four cue-presentation methods were randomized in blocks of four sessions. Two random orders of the cue-presentation methods were produced. One order was used for 4 birds, and the second order was used for the remaining 4 birds. Regardless of the specific cue-presentation method, all test sessions consisted of $120 \mathrm{CC}$ trials and 24 probe trials. Probe trials consisted of $8 \mathrm{CC}$ trials, $8 \mathrm{IC}$ trials, and $8 \mathrm{NC}$ trials. One probe trial occurred randomly within each block of $5 \mathrm{CC}$ trials. The probability of reinforcement for correct responses on probe trials was the same as that on CC trials. All other aspects of the test sessions were as described previously. A total of 32 test sessions were given.

\section{Results and Discussion}

The data from probe trials averaged over subjects and test sessions are shown in Figure 2. An analysis of variance revealed significant effects of trial type $[F(2,14)=14.57$, $p<.001]$ and cue-presentation condition $[F(3,21)=4.05$, $p<.05]$ and a significant interaction $[F(6,42)=5.53$, $p<.001]$. A simple main-effects analysis indicated a significant effect of trial type whenever the cue was superimposed on the sample $[F(2,14)=20.27,8.57,15.32]$ but not when the cue was presented after the sample $(F<1)$. Accuracy on CC and NC trials was the same regardless of whether the cue was superimposed on the sample or presented after the sample. However, accuracy on IC trials was significantly lower when the cue was superimposed on the sample than when it followed the sample $[F(3,21)=$ $8.04, p<.001]$. The critical variable in producing cuing effects appeared to be the superimposition of the cue on the sample stimulus. Presentation of the cue during all or part of the DI did not appear to affect the results.

\section{EXPERIMENT 3}

In the previous experiment, the decrement in accuracy on IC trials was a function only of whether the cue was superimposed on the sample. Postsample cue presentation was ineffective. In Experiment 3, the effect of providing the cue prior to the sample was examined.

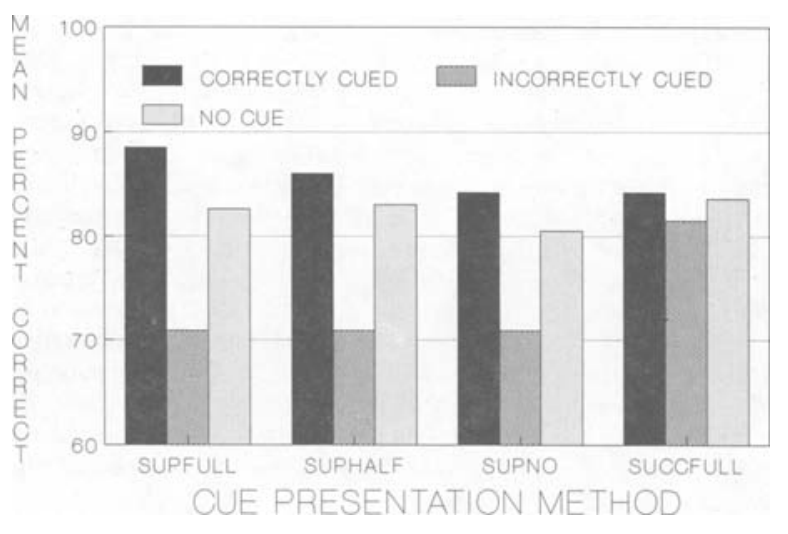

Figure 2. Mean percentage of correct matching responses during testing as a function of trial type (correct, incorrect, or no cue) and cue-presentation condition in Experiment 2.

\section{Method}

Subjects and Apparatus. The subjects and the apparatus were the same as in the previous experiments.

Procedure. After some additional testing that proved to be uninformative, the birds were given 31 sessions of baseline training similar to those given in the previous experiment. The cue was superimposed on the sample, and the first keypeck after $5 \mathrm{sec}$ terminated both stimuli. The DI was gradually increased over sessions to $5 \mathrm{sec}$. One bird could not sustain accurate performance with the 5-sec DI, and it was dropped from the study. The remaining 7 birds all averaged $74 \%$ correct or higher over the last 5 sessions of training with the 5 -sec DI.

During the testing phase, the birds were exposed to three methods of cue presentation on probe trials. In the presample cue-sample alone condition, the cue was presented by itself for $5 \mathrm{sec}$ following a peck to the warning light. The cue was terminated, and the sample was presented by itself for $5 \mathrm{sec}$. Following offset of the sample, a 5-sec DI occurred with no stimuli presented on the keys. In the no presample cue-superimposed compound condition, a peck to the warning light resulted in presentation of the cue superimposed on the sample for $5 \mathrm{sec}$. Following offset of the superimposed compound, a 5-sec DI occurred with no stimuli presented on the keys. In the postsample cue-sample alone condition, a peck to the warning light resulted in presentation of the sample for $5 \mathrm{sec}$. Following offset of the sample, the cue was presented during the 5-sec DI. During testing, the three cue-presentation methods were randomized in blocks of three sessions. Two random orders of cue-presentation methods were produced. One order was used for 4 birds, and the second order was used for the 3 remaining birds. Unlike in Experiment 2, in this study the different cue-presentation methods occurred only on probe trials. The majority of trials during a test session were like those of baseline training sessions.

Each test session consisted of $120 \mathrm{CC}$ trials and 24 probe trials. Probe trials consisted of $8 \mathrm{CC}$ trials, $8 \mathrm{IC}$ trials, and $8 \mathrm{NC}$ trials. One probe trial occurred randomly within each block of $5 \mathrm{CC}$ trials. The probability of reinforcement for correct responses on probe trials was the same as that on CC trials. All other aspects of the test sessions were as described for Experiment 2. A total of 32 test sessions were given, 8 for each of the three cue-presentation conditions.

\section{Results and Discussion}

The data from probe trials averaged over subjects and test sessions are shown in Figure 3. An analysis of variance revealed an effect of trial type $[F(2,12)=13.53$, $p<.001]$ and a significant interaction of trial type and cue-presentation condition $[F(4,24)=3.99, p<.05]$. When the cue was presented prior to the sample $[F(2,12)$ $=1.57, p>.05]$ or after the sample $[F(2,12)=3.62$, $p>.05]$, there was no cuing effect. However, there was a strong cuing effect when the cue was superimposed on the sample stimulus $[F(2,12)=19.49, p<.001]$. A Newman-Keuls test indicated that when the cue was superimposed on the sample, accuracy was significantly greater on CC and NC trials than on IC trials. These data are consistent with those presented earlier. Robust cuing effects were obtained when the cue was superimposed on the sample but not when it occurred either before or after the sample on probe trials.

Additional analyses also indicated that accuracy on the high-probability-of-reinforcement dimension was greater than on the low-probability-of-reinforcement dimension when the test dimension was correctly cued and the cue was superimposed on the sample $[F(1,6)=10.25, p<.05]$. Although there were similar trends for the NC and IC conditions, none of these were significant except for the NC data in the postsample cue condition $[F(1,6)=6.27, p<.05]$. 

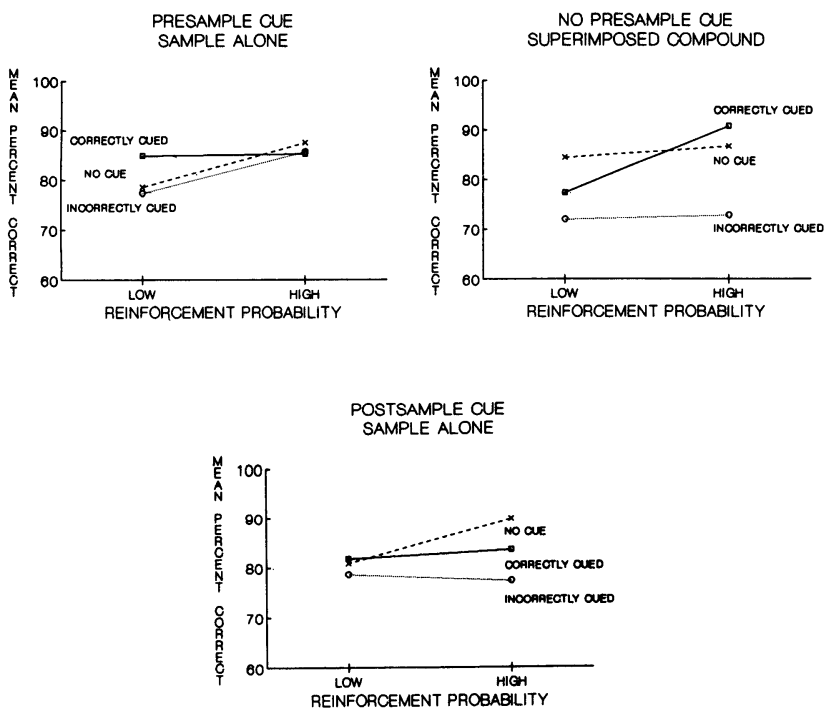

Figure 3. Mean percentage of correct matching responses during testing as a function of trial type (correct, incorrect, or no cue), reinforcement probability, and cue-presentation condition in Experiment 3.

\section{GENERAL DISCUSSION}

The results produced by manipulating the method of cue presentation can be summarized rather easily. In all three experiments, when comparison dimension cues were superimposed on the sample, significant effects of cuing on matching accuracy were observed. Specifically, accuracy was lower on IC trials than on CC or NC trials. In addition, Experiment 3 revealed that on CC trials accuracy was higher on the comparison dimension associated with the higher probability of reinforcement. When comparison dimension cues were presented only either before or after the sample stimulus, there were no significant cuing effects on overall matching accuracy and no reliable differences as a function of reinforcement probability.

When presented as presample cues, comparison dimension cues do not appear to be used to selectively encode the sample stimulus. If they were used in this way, incorrectly cuing the comparisons should have significantly reduced accuracy in the presample cue condition. When presented as postsample cues, comparison dimension cues do not appear to encourage selective rehearsal of a particular response decision. If they did act in this way, incorrectly cuing the comparisons should have significantly lowered accuracy in the postsample cue condition. In addition, the results of Experiment 1 were inconsistent with the prediction of the dual coding model that IC trials would be more affected by increases in DI duration. This result has also been reported by Grant and MacDonald (1990).

In light of these findings, the validity of using comparison dimension cuing effects to address the issue of prospective versus retrospective processing must be reevaluated. The results suggest that the sample and superimposed cues are not decomposed by pigeons but that the cues are perceived and processed as a unitary configuration. For example, the red-triangle compound is coded as "peck red," the red-circle compound is coded as "peck vertical," and so forth. Considering the training of these birds in a previous study (Santi et al., 1988) and in the current series of experiments, the birds could also have learned response codes for element samples (i.e., NC trials). For example, red is coded as "peck red or vertical," and green is coded as "peck green or horizontal." On IC trials, the sample and superimposed cues would be inappropri- ate for the comparisons presented. However, through processes of stimulus generalization, this compound should be more similar to one other compound or element sample than to the others. For example, the redtriangle compound is more similar to either the red-circle compound or red than it is to green-triangle, green-circle, or green. This should result in activation of the correct response code with a probability greater than zero. Consequently, matching accuracy on IC trials would be expected to be above chance but less than that observed on CC or NC trials. When the processes of stimulus generalization operate during the test phase of a trial, the interpretation outlined here is the same as that originally proposed by D'Amato (cited in Honig \& Thompson, 1982). When they operate during the sample-presentation phase of a trial, the interpretation is the same as that developed by Grant and MacDonald (1990) to account for comparison cuing effects. The present data could equally well be accounted for in terms of retrospective coding or prospective coding of sample-cue compounds.

The data showing greater accuracy on the high-probability-ofreinforcement dimension than on the low-probability-of-reinforcement dimension can be explained in terms of differential attention to sample stimuli when the dimension is correctly cued. This effect was reliably obtained only when the comparison dimension was correctly cued and the cue was superimposed on the sample. Under these conditions, the birds could attend more to the compound samples signaling the highprobability-of-reinforcement dimension and, as a result, display greater accuracy on these trials.

In summary, cues that were superimposed on the sample stimulus and that signaled different comparison dimensions and reinforcement probabilities were not analytically separated from the sample. Rather, the sample-cue compound appeared to be processed and perceived as a unitary configuration, and the cues individually provided no information when presented prior to or after the sample stimulus.

\section{REFERENCES}

Cohen, J. S., Galgan, R., \& Fuerst, D. (1986). Retrospective and prospective short-term memory in delayed response tasks in rats. Animal Learning \& Behavior, 14, 38-50.

Grant, D. S. (1982). Prospective versus retrospective coding of samples of stimuli, responses, and reinforcers in delayed matching with pigeons. Learning \& Motivation, 13, 265-280.

Grant, D. S., \& MACDonald, S. E. (1990). An evaluation of the role of dual coding in mediating the effect of incorrectly cuing the comparison dimension in delayed matching in pigeons. Animal Learning \& Behavior, 18, 151-156.

HoNig, W. K., \& Thompson, R. K. R. (1982). Retrospective and prospective processing in animal working memory. In G. H. Bower (Ed.), The psychology of learning and motivation: Advances in research and theory (Vol. 16, pp. 239-283). New York: Academic Press.

Kraemer, P. J., \& Roberts, W. A. (1984). Short-term memory for visual and auditory stimuli in pigeons. Animal Learning \& Behavior, 12, 275-284.

Santi, A., Musgrave, S., \& Bradford, S. A. (1988). Utilization of cues signaling different test stimulus dimensions in delayed matching to sample by pigeons. Learning \& Motivation, 19, 87-98.

SANTI, A., \& RoberTs, W. A. (1985). Prospective representation: The effects of varied mapping of sample stimuli to comparison stimuli and differential trial outcomes on pigeons' working memory. Animal Learning \& Behavior, 13, 103-108.

Stonebraker, T. B., \& Rilling, M. (1984). Retrospective versus prospective processes in delayed matching to sample. Bulletin of the Psychonomic Society, 22, 372-375.

Urcuioli, P. J., \& Zentall, T. R. (1986). Retrospective coding in pigeons' delayed matching-to-sample. Journal of Experimental Psychology: Animal Behavior Processes, 12, 69-77.

(Manuscript received December 3, 1990.) 